\title{
Performance of compressed air energy storage system with regenerative heat exchangers
}

\author{
Shibiao Wang ${ }^{1}$, Wei Liang $^{1}, X i$ Lai $^{1}$, and Wenqiang Sun $^{1,2^{*}}$ \\ ${ }^{1}$ Department of Thermal Engineering, Northeastern University, Shenyang 110819, China \\ ${ }^{2}$ SEP Key Laboratory of Eco-Industry, Ministry of Ecology and Environment, Shenyang 110819, China
}

\begin{abstract}
In order to improve the heat storage and heat exchange system of advanced adiabatic compressed air energy storage (AA-CAES) system, an AA-CAES system with regenerative heat exchangers (RHEs) is studied. The RHE is used to replace the conventional complex units, including heat exchangers, high temperature tank, and low temperature tank mode. For the AA-CAES with RHEs, the energy storage system is simplified to reduce the heat loss in the heat exchange and storage processes, and thus, the output work, energy storage density, energy storage efficiency of the system are improved. The thermodynamic model is established and the influences of compression ratio distribution, expansion ratio distribution and ambient temperature on the system performance are investigated. The results show that for the AA-CAES with RHEs, when the ratio of compression ratios is 1.14 , the input work of the compressor is the minimum, the energy storage efficiency is $66.42 \%$, and the energy storage density is $3.61 \mathrm{kWh} / \mathrm{m}^{3}$. When the ratio of expansion ratios is 0.82 , the energy storage efficiency reaches the maximum value of $67.38 \%$, and the energy storage density reaches the maximum value of $3.66 \mathrm{kWh} / \mathrm{m}^{3}$.
\end{abstract}

\section{Introduction}

With the continuous development and utilization of renewable energy such as intermittent solar energy and wind energy, the stability of power grid is affected [1-3]. To address this issue, electric energy storage technologies emerge in recent years, playing an important role in the stability of the grid [4]. Among the energy storage technologies, the compressed air energy storage (CAES) technology stands out for its large capacity, low cost, long service life, no geographical constraints and technology readiness level [5]. During the storage period, CAES system consumes electric energy to compress air into the air storage cavern; and during the energy release period, the high-pressure air in the cavern drives expanders to generate electricity [1]. Advanced adiabatic compressed air energy storage (AA-CAES) system integrates heat exchangers and thermal storage tanks to conventional CAES systems [6]. Using heat generated during the compression process to heat air at the expander inlet, the utility model is less dependent on fossil fuels, has higher efficiency and is more environmentally friendly [7]. However, AA-CAES generally includes heat exchangers, high-temperature tank and low-temperature tank $[6,7]$. The complicated structure brings many problems, such as heat loss, large occupied area and high investment cost.

In order to solve these problems, an AA-CAES system with regenerative heat exchangers (RHEs) is studied in this paper. The RHEs are used to replace the conventional heat exchangers, high temperature tank and low temperature tank, aiming at simplifying the system structure and improving the system performance.

\section{System design}

The AA-CAES system with RHEs is shown in Fig. 1. The main components include compressors, RHEs, air storage cavern, expanders, etc.

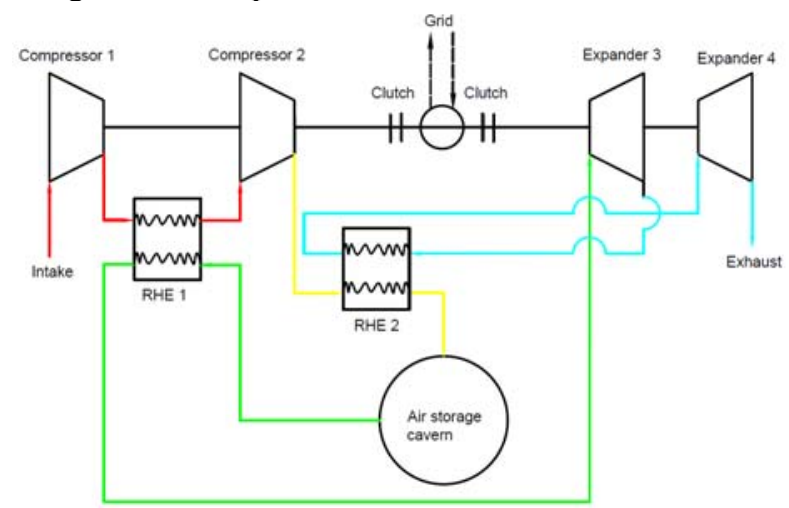

Fig. 1. Schematic diagram of AA-CAES system with RHEs.

During the energy storage period, air enters the compressors 1 and 2 for adiabatic compression, and the temperature and pressure of air rise rapidly. Then the high-temperature and high-pressure air enters the RHEs 1 and 2 to release heat and reduce the temperature. High-pressure air after two compression and heat exchange processes is stored in the air storage cavern. During the energy release period, high-pressure air from the cavern enters RHE 1 to absorb heat and increase the

\footnotetext{
*Corresponding author: sunwq@mail.neu.edu.cn
} 
temperature. The preheated high-pressure air enters expanders 1 and 2 to generate electricity. The use of RHEs can simplify the AA-CAES system and reduce the heat loss in the process of heat storage. Cordierite is selected as the heat storage material filled in RHEs because of its good fire resistance and low thermal expansion rate, so as to improve the expander inlet temperature and the resultant system efficiency.

\section{Thermodynamic model}

The thermodynamic model is established based on the following assumptions:

(i) The system is in steady state and air is considered as the ideal gas.

(ii) The pressure losses of air in pipeline, RHEs and air storage cavern are ignored.

(iii) The compression and expansion are regarded as adiabatic processes, and there is no heat exchange with the surroundings.

(iv) The heat losses of RHEs and pipeline are ignored.

\subsection{Compressor}

For an ideal state, the compression process of air in compressors is adiabatic. In fact, taking compressor 1 as an example, compression leads to entropy increase, which is assumed to be a polytropic process, and the polytropic index is set to $n_{1}$. Let the compression ratio of compressor 1 be:

$$
\beta_{1}=\frac{p_{1, \mathrm{out}}}{p_{1, \mathrm{in}}}
$$

The adiabatic efficiency of compressor 1 is:

$$
\eta_{\mathrm{c}, 1}=\frac{\beta_{1}^{\frac{k-1}{k}}-1}{\beta_{1}^{\frac{n_{1}-1}{n_{1}}}-1}
$$

where $p_{1, \text { in }}$ and $p_{1, \text { out }}$ are air pressure at the inlet and outlet of compressor 1 , respectively, $\mathrm{Pa}$; $k$ is the adiabatic index of air.

The inlet temperature is set to $T_{1 \text {,in, }}$ i.e., the ambient temperature. The air temperature at the outlet of compressor 1 is:

$$
T_{1, \text { out }}=T_{1, \text { in }} \beta_{1}^{\frac{n_{1}-1}{n_{1}}}
$$

When air flows through compressor 1 , the consumed compression work can be calculated as follows:

$$
w_{\mathrm{c}, 1}=\frac{c_{p}\left(T_{1, \mathrm{out}}-T_{1, \mathrm{in}}\right)}{\eta_{\mathrm{c}, 1}}
$$

where $c_{p}$ is the specific heat capacity of air at constant pressure, $\mathrm{J} /(\mathrm{kg} \cdot \mathrm{K})$.

Likewise, the temperature of air at the outlet of compressor 2 is:

$$
T_{2, \text { out }}=T_{2, \text { in }} \beta_{2}^{\frac{n_{2}-1}{n_{2}}}
$$

where $n_{2}$ is the polytropic index of compressor 2 .

The consumed compression work of compressor 2 is:

$$
w_{\mathrm{c}, 2}=\frac{c_{p}\left(T_{2, \mathrm{out}}-T_{2, \mathrm{in}}\right)}{\eta_{\mathrm{c}, 2}}
$$

\subsection{Regenerative heat exchanger}

In the heat exchange and energy storage processes, the high-temperature and high-pressure air from compressor 1 enters RHE 1 to release heat. $\varepsilon_{1}$ is the effectiveness of RHE 1, which is the ratio of the temperature drop of air passing through the heat exchanger to the temperature difference between of outlet air and inlet flue gas, i.e.,

$$
\varepsilon_{1}=\frac{T_{1, \text { out }}-T_{1, \text { out }-\mathrm{s}}}{T_{1, \text { out }}-T_{\text {flu } 1}}
$$

where $T_{\text {flu1 }}$ is the temperature of heat exchange fluid at the inlet of RHE $1, \mathrm{~K} ; T_{1, \text { out-s }}$ is the air temperature flowing out of RHE 1 after heat exchange and is expressed as:

$$
T_{1, \text { out }-\mathrm{s}}=\left(1-\varepsilon_{1}\right) T_{1, \mathrm{out}}+\varepsilon_{1} T_{\text {flu1 } 1}
$$

Heat storage material in RHE 1 absorbs heat:

$$
q_{1}=c_{p}\left(T_{1, \mathrm{out}}-T_{1, \mathrm{out}-\mathrm{s}}\right)
$$

The temperature of compressed air at the outlet of RHE 2 is:

$$
T_{2, \text { out }-\mathrm{s}}=\left(1-\varepsilon_{2}\right) T_{2, \text { out }}+\varepsilon_{2} T_{\text {flu2 }}
$$

where $T_{\text {flu2 }}$ is the temperature of heat exchange fluid at the inlet of RHE 2, K; $T_{2 \text {,out-s }}$ is the air temperature flowing out of RHE 2 after heat exchange.

Heat storage material in RHE 2 absorbs heat:

$$
q_{2}=c_{p}\left(T_{2, \text { out }}-T_{2, \text { out }-\mathrm{s}}\right)
$$

During the heat exchange and energy release periods, the high-pressure air in the air storage cavern first enters the RHE to absorb heat, and then enters the expander to do work.

Heat absorbed by unit mass of high-pressure air in RHEs 1 and 2 are respectively expressed as:

$$
\begin{aligned}
& q_{3}=c_{p}\left(T_{\text {flu1-s }}-T_{\text {flu1 } 1}\right) \\
& q_{4}=c_{p}\left(T_{\text {flu2-s }}-T_{\text {flu2 }}\right)
\end{aligned}
$$

\subsection{Expander}

Ideally, the expansion process of air in expanders is adiabatic. Taking expander 1 as an example. Compared with the compression process, the adiabatic efficiency of the expander is:

$$
\eta_{\mathrm{e}}=\frac{1-\beta_{3}^{\frac{n_{3}-1}{n_{3}}}}{1-\beta_{3}^{\frac{k-1}{k}}}
$$

where $n_{3}$ is the polytropic index of the expansion process; $\beta_{3}$ is the ratio of expansion ratios of expander 1 and is expressed as:

$$
\beta_{3}=\frac{p_{3, \mathrm{out}}}{p_{3, \text { in }}}
$$

where $p_{3 \text {,in }}$ and $p_{3, \text { out }}$ are the inlet and outlet air pressure of expander $1, \mathrm{~Pa}$.

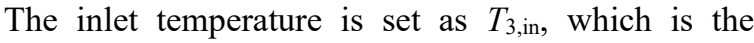
temperature of the high-pressure air flowing out of RHE 1. The air temperature at the outlet of expander 1 is:

$$
T_{3, \text { out }}=T_{3, \text { in }} \beta_{3}^{\frac{n_{3}-1}{n_{3}}}
$$

When air flows through expander 1 , the axial work generated is:

$$
w_{\mathrm{e}, 1}=c_{p}\left(T_{3, \text { in }}-T_{3, \mathrm{out}}\right) \eta_{\mathrm{e}, 1}
$$

Likewise, let the expansion ratio of expander 2 be: 


$$
\beta_{4}=\frac{p_{4, \mathrm{out}}}{p_{4, \mathrm{in}}}
$$

where $p_{4, \text { in }}$ and $p_{4, \text { out }}$ are the inlet and outlet air pressure of expander $2, \mathrm{~Pa}$.

The inlet temperature is set as $T_{4, \text { in }}$, which is the temperature of the heat exchange high-pressure air flowing out of RHE 2. The air temperature at the outlet of expander 2 is:

$$
T_{4, \text { out }}=T_{4, \text { in }} \beta_{4}^{\frac{n_{4}-1}{n_{4}}}
$$

where $n_{4}$ is the polytropic index of expander 2 .

For expander 2, the axial work generated is:

$$
w_{\mathrm{e}, 2}=c_{p}\left(T_{4, \mathrm{in}}-T_{4, \mathrm{out}}\right) \eta_{\mathrm{e}, 2}
$$

\subsection{System evaluation indices}

Energy storage efficiency is defined as the ratio of output power to input power and is expressed as:

$$
\eta=\frac{m_{\mathrm{e}}\left(w_{\mathrm{e}, 1}+w_{\mathrm{e}, 2}\right) \tau_{\mathrm{e}}}{m_{\mathrm{c}}\left(w_{\mathrm{c}, 1}+w_{\mathrm{c}, 2}\right) \tau_{\mathrm{c}}}
$$

where $m_{\mathrm{c}}$ and $m_{\mathrm{e}}$ are the flow rates of air passing through compressors and expanders, respectively; $\tau_{\mathrm{c}}$ and $\tau_{\mathrm{e}}$ are the time of energy storage and release periods, respectively.

Energy storage density is defined as the ratio of expansion work to the volume of the air storage cavern and expressed as:

$$
D=\frac{w_{\mathrm{e}}}{V}
$$

where $V$ is the volume of the air storage cavern.

\section{Results and analysis}

The original system parameters are shown in Table 1. The thermophysical properties of the heat storage material cordierite are shown in Table 2.

\subsection{Compression ratio distribution}

The ratio of compression ratios is defined as:

$$
r_{\mathrm{c}}=\beta_{1} / \beta_{2}
$$

Fig. 2 shows the relationship between compressor input work, expander output work and the ratio of compression ratios. With the increasing ratio of compression ratios, the total input work of the compressors decreases first and then increases slowly, and reaches the minimum value, which is $543.63 \mathrm{MWh}$, at the compression ratio of 1.14 . This is because the air temperature at the inlet of compressor 1 is lower than that at the inlet of compressor 2. When the ratio of compression ratios is equal to 1.14 , the compression ratio of compressor 1 is greater than that of compressor 2. In addition, the air temperature at the outlet of the two compressors is equal, and the total input work of the two compressors is the smallest. The total output work of the expander decreases with the increase in the ratio of compression ratios, but the change is not clear if the ratio of compression ratios is greater than 1 . This is because the total output work of the two expanders is mainly related to the temperature and flow rate of the air flowing through the RHEs.
Table 1. System parameters.

\begin{tabular}{|c|c|c|c|}
\hline Parameters & Symbols & Units & Values \\
\hline $\begin{array}{c}\text { maximum pressure of air } \\
\text { cavern }\end{array}$ & $p_{\max }$ & $\mathrm{MPa}$ & 6.5 \\
\hline $\begin{array}{c}\text { minimum pressure of air } \\
\text { cavern }\end{array}$ & $p_{\text {min }}$ & $\mathrm{MPa}$ & 3.5 \\
\hline air storage cavern volume & $V$ & $\mathrm{~m}^{3}$ & 100000 \\
\hline $\begin{array}{c}\text { ambient temperature } \\
\text { adiabatic efficiency of } \\
\text { compressor }\end{array}$ & $T_{0}$ & ${ }^{\circ} \mathrm{C}$ & 20 \\
\hline $\begin{array}{c}\text { adiabatic efficiency of } \\
\text { expander }\end{array}$ & $\eta_{\mathrm{e}}$ & - & 0.85 \\
\hline
\end{tabular}

Table 2. Thermal properties of cordierite.

\begin{tabular}{|c|c|c|}
\hline Parameters & Values & Units \\
\hline melting temperature & 1450 & ${ }^{\circ} \mathrm{C}$ \\
\hline hole wall density & 1.6 & $\mathrm{~g} / \mathrm{m}^{3}$ \\
\hline coefficient of thermal expansion & $10^{-6}$ & $1 /{ }^{\circ} \mathrm{C}$ \\
\hline $\begin{array}{c}\text { thermal conductivity at room } \\
\text { temperature }\end{array}$ & $9.2 \times 10^{-3}$ & $\mathrm{~W} /\left(\mathrm{cm} \cdot{ }^{\circ} \mathrm{C}\right)$ \\
\hline $\begin{array}{c}\text { specific heat capacity at room } \\
\text { temperature }\end{array}$ & 840 & $\mathrm{~J} /\left(\mathrm{kg} \cdot{ }^{\circ} \mathrm{C}\right)$ \\
\hline $\begin{array}{c}\text { compressive strength } \\
\text { channel density }\end{array}$ & $1.24 \times 10^{7}$ & $\mathrm{~N} / \mathrm{m}^{3}$ \\
\hline $\begin{array}{c}\text { hem } \\
\text { che }\end{array}$ & $1.37 \times 10^{6}$ & $\mathrm{~N} / \mathrm{m}^{3}$ \\
\hline
\end{tabular}

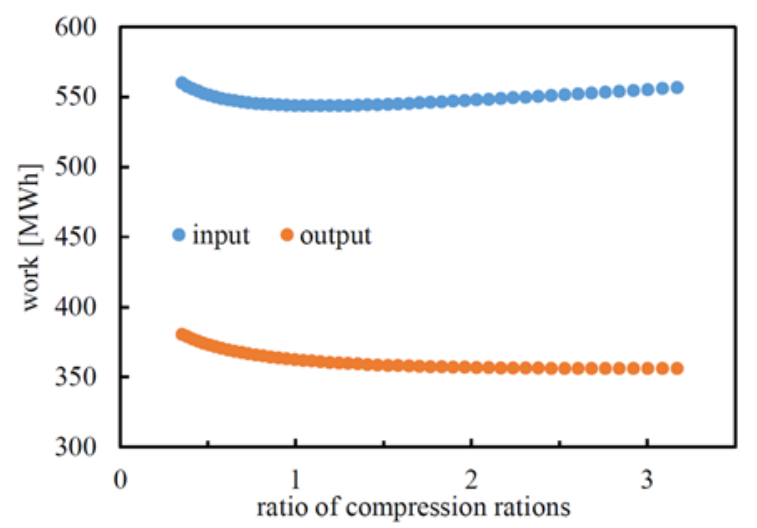

Fig. 2. Relationship between compressor input work, expander output work and compression ratio distribution.

Fig. 3 shows the relationship between energy storage efficiency and the ratio of compression ratios. It can be seen that the energy storage efficiency decreases as the ratio of compression ratios increases. Before the total input work of the compressors reaches the minimum value, the total output work of the expanders is decreasing, resulting in a decrease in the energy storage efficiency. After the total input work of the compressor reaches the minimum value, the total output work of the expanders remains basically unchanged, but the total 
input work of compressors gradually increases, resulting in the continued decline of energy storage efficiency.

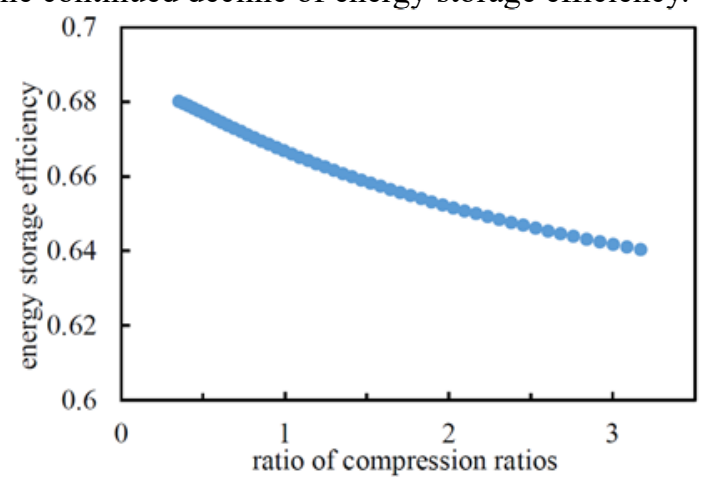

Fig. 3. Relationship between energy storage efficiency and compression ratio distribution.

Fig. 4 shows the relationship between the energy storage density and the ratio of compression ratios. The energy storage density decreases with the increasing ratio of compression ratios. When the input work of compressors is the smallest, the ratio of compression ratios is 1.14 , the energy storage efficiency is $66.42 \%$, and the energy storage density is $3.61 \mathrm{kWh} / \mathrm{m}^{3}$.

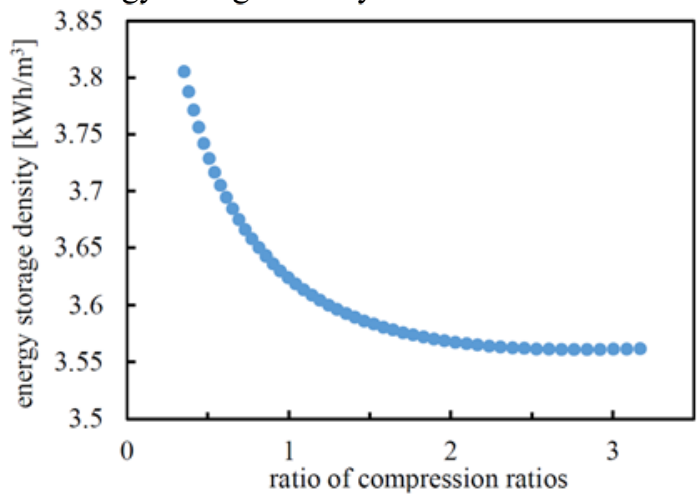

Fig. 4. Relationship between energy storage density and compression ratio distribution.

\subsection{Expansion ratio distribution}

The ratio of expansion ratio is defined as:

$$
r_{\mathrm{c}}=\beta_{3} / \beta_{4}
$$

Fig. 5 shows the relationship between the input work of the compressors, the output work of the expanders and the ratio of expansion ratios. It can be seen that with the increasing ratio of expansion ratios, the total output work of the expanders firstly increases and then decreases, but the reduction is slight. When the expansion ratio is 0.82 , the total output of the expanders reaches its maximum, which is $366.30 \mathrm{MWh}$.

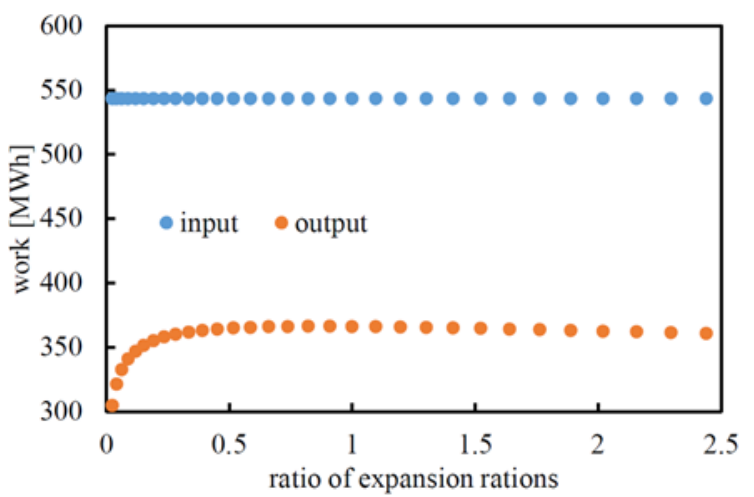

Fig. 5. Relationship between input work of compressors, output work of expanders and expansion ratio distribution.

Figs. 6 and 7 show the relationship between the energy storage efficiency and the ratio of expansion ratios, and the relationship between the energy storage density and the ratio of expansion ratios, respectively. It can be seen that the profiles of energy storage efficiency and energy storage density are roughly the same as the change in expander output work. They all increase rapidly first and then decrease slowly as the ratio of expansion ratios increases. When the ratio of expansion ratios increases to 0.82 , the energy storage efficiency reaches the maximum value of $67.38 \%$, and the energy storage density reaches the maximum value of 3.66 $\mathrm{kWh} / \mathrm{m}^{3}$. The main reason is that the total output work of the expanders increases first and then decreases with increasing ratio of expansion ratios.

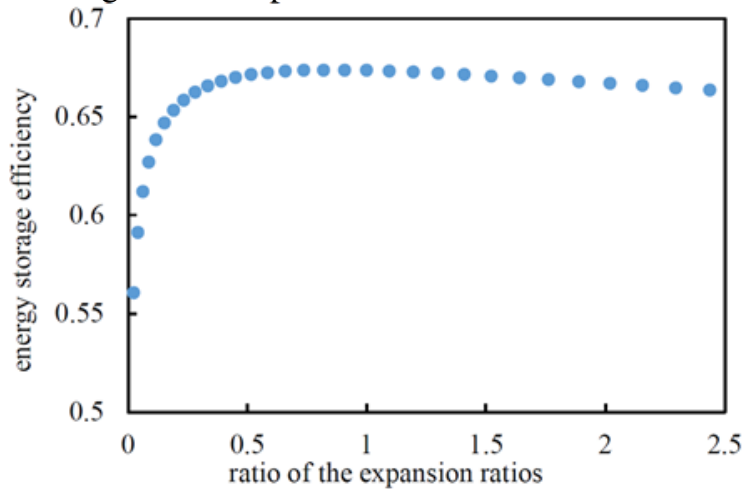

Fig. 6. Relationship between energy storage efficiency and the expansion ratio distribution.

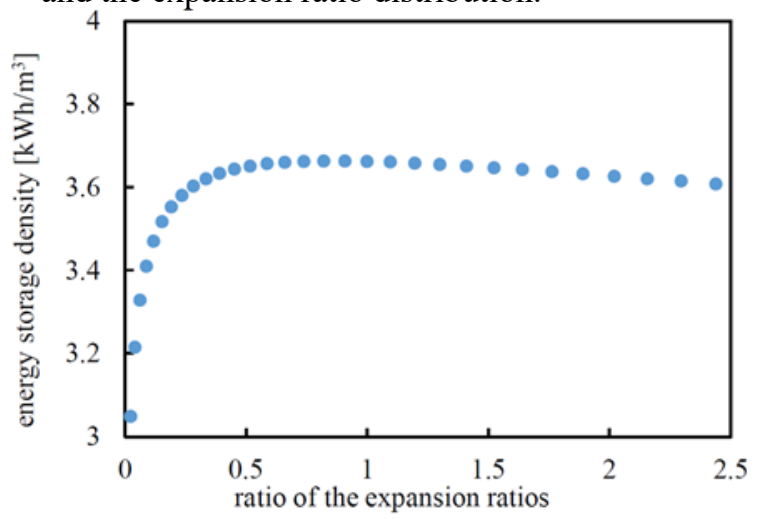

Fig. 7. Relationship between energy storage density and the expansion ratio distribution. 


\subsection{Ambient temperature}

Fig. 8 shows the relationship between the input work of compressors, the output work of expanders and the ambient temperature. The total input work of compressors and the total output work of expanders both decrease with the increase in ambient temperature. The increase in environment temperature will cause the increase in the temperature at the inlet and outlet of compressors and expanders. In addition, the increase in ambient temperature will cause an increase in the temperature of high-pressure air in the air storage cavern. In the cavern with constant pressure, the increase in air temperature leads to the decrease in mass and further leads to the decrease in the total input work of the compressors and the total output work of the expanders. Because air quality has a greater influence, both the total input work of compressors and the total output work of expanders decrease.

Fig. 9 shows the relationship between energy storage efficiency and ambient temperature. The energy storage efficiency of the system slowly decreases as the ambient temperature increases. This is because the total input work of compressors and the total output work of expanders decrease with the increase in ambient temperature, but the change of the total output work outweighs the change in the total input work. Therefore, the energy storage efficiency of the system decreases slowly with the increase in ambient temperature.

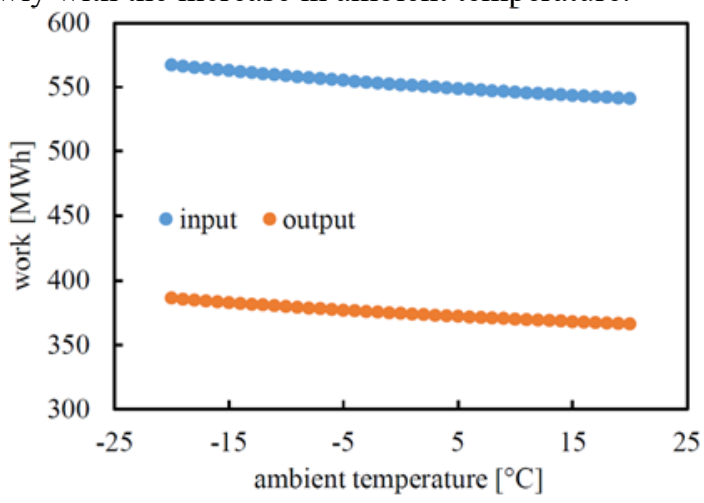

Fig. 8. Relationship between input work of compressors, output work of expanders and ambient temperature.

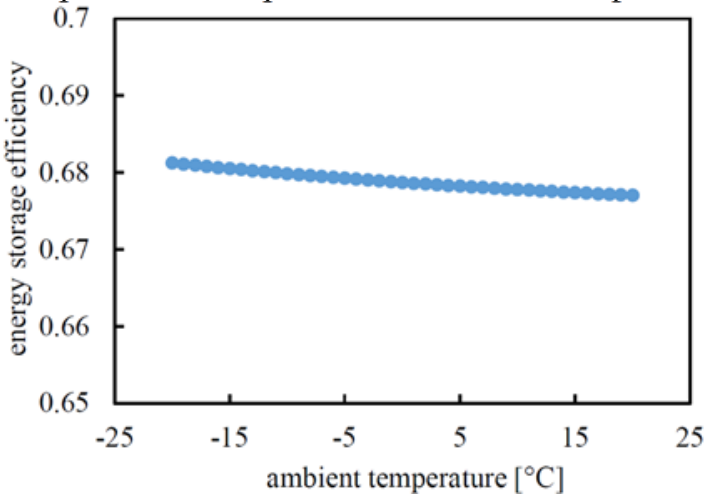

Fig. 9. Relationship between energy storage efficiency and ambient temperature.

Fig. 10 shows the relationship between energy storage density and ambient temperature. As can be seen, the energy storage density of the system decreases with the increase in ambient temperature. The reason is that the increase in the ambient temperature causes the decrease in the total output work of the expanders and finally leads to the decrease in the energy storage density.

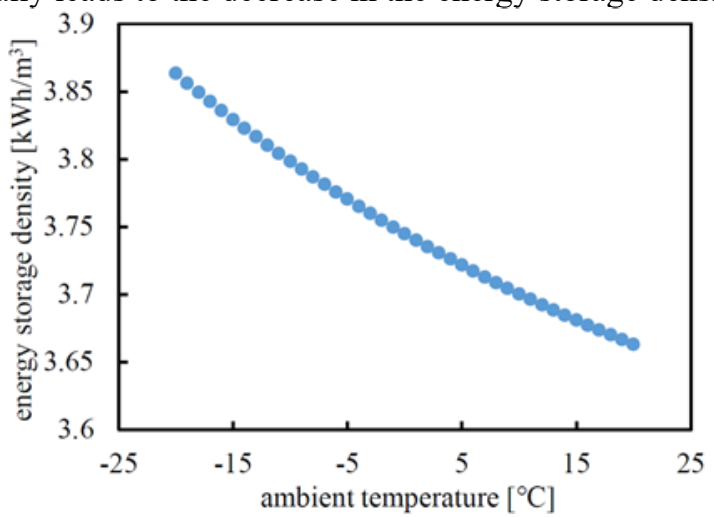

Fig. 10. Relationship between energy storage density and ambient temperature.

It can be concluded that the lower the ambient temperature, the higher the energy storage efficiency and the higher the energy storage density of the system. Taking $0{ }^{\circ} \mathrm{C}$ as an example, the energy storage efficiency of the system is $67.87 \%$, and the energy storage density is $3.74 \mathrm{kWh} / \mathrm{m}^{3}$.

\section{Conclusion}

In this paper, the AA-CAES system with RHEs is proposed to replace conventional heat exchangers, high-temperature and low-temperature tanks.

For the AA-CAES system using RHEs, as the ratio of compression ratios increases, the system energy storage efficiency and energy storage density gradually decrease. When the ratio of compression ratios is 1.14 , the compressor input work of the system is minimum, the energy storage efficiency reaches $66.42 \%$, and the energy storage density is $3.61 \mathrm{kWh} / \mathrm{m}^{3}$.

As the ratio of expansion ratios increases, the energy storage efficiency and energy storage density of the system first increase and then decrease. When the ratio of expansion ratios is 0.82 , the energy storage efficiency reaches the maximum of $67.38 \%$, and the energy storage density reaches the maximum of $3.66 \mathrm{kWh} / \mathrm{m}^{3}$.

With the increase in ambient temperature, the energy storage efficiency and density of the system decrease. The lower the ambient temperature, the higher the energy storage efficiency and energy storage density.

\section{Acknowledgement}

This work was supported by the National Training Program of Innovation and Entrepreneurship for Undergraduates (202010145041) and the Fundamental Research Funds for the Central Universities (N182410001, N2025022), and the National Natural Science Foundation of China (51734004).

\section{References}

1 P. An, North China Elec. Power Univ. (2018) 
2 Q. Xu, Y. Sun, L. Liu, et al., Zhejiang Elec. Power 385 (2019) 3-10

3 J. Zhang, S. Zhou, S. Li, et al., New Energy Prog. 62 (2018) 60-70

4 S. Guo, North China Elec. Power Univ. (2019)

5 Z. Han, S. Liu, Q. Zhou, Y. Pang, Proc. Chin. Soc. Elec. Eng. 6 (2016) 3373-3380

6 Z. Han, Q. Zhou, Y. Wang, et al., Acta Sol. Energy 3 (2016) 629-635

7 S. Pang, North China Elec. Power Univ. (2019) 